Z. klin. Chem. u. klin. Biochem.

9. Jg., S. $508-515$, November 1971

\title{
Die quantitative Serumhaptoglobinbestimmung mit der Peroxydaseaktivierungsmethode und der radialen Immunodiffusion. Ein Methodenvergleich
}

\author{
Von H. J. Braun und F. W. AlY
}

\begin{abstract}
Aus der Medizinischen Klinik (Direktor: Prof. Dr. Dr. b. c. H. E. Bock) und der Abteilung Innere Medizin II
(Vorstand: Prof. Dr. H. D. Waller) der Universität Tübingen
\end{abstract}

(Eingegangen am 9. Juli 1971)

Für die quantitative Haptoglobinbestimmung im Serum wurde die Peroxydaseaktivierungsmethode nach JAYLE mit der radialen Immunodiffusion nach MANCrNI und Mitarbeitern verglichen. Der methodische Fehler beider Verfahren erwies sich als etwa gleich groß. Der statistische Vergleich der mit beiden Methoden ermittelten Werte ergab signifikante Korrelationen für die 3 genetischen Haptoglobin-Typen und für jeden Typ eine eigene Regressionsgerade.

Mit der immunologischen Methode wurden höhere Serumwerte gemessen als mit dem Peroxydaseverfahren: Diese Beobachtung konnte auf Unterschiede in den Bezugssystemen zwischen der immunologisch bestimmten Proteinkonzentration und der mit der Peroxydasemethode ermittelten Hämoglobinbindungskapazität zurückgeführt werden. Für zusätzliche typenabhängige Differenzen wurde ein unterschiedliches Hämoglobinbindungsverhältnis der monomeren und polymeren Formen angenommen. Zur Umrechnung der immunologisch bestimmten Haptoglobinkonzentration in Hämoglobinbindungskapazität (und umgekehrt) wurden die entsprechenden Regressionsgleichungen angegeben.

Hämoglobinzusatz zum Serum führt beim Peroxydaseverfahren zur Ermittlung zụ niedriger Haptoglobinwerte, während die immunologisch gemessenen Haptoglobinkonzentrationen zu hoch liegen.

Für die 3 genetischen Haptoglobin-Typen ließen sich mit beiden Methoden verschiedene Notmwertbereiche aufstellen. Wegen des unterschiedlichen Verhaltens der 3 genetischen Haptoglobin-Typen in der radialen Immunodiffusion ist die Kenntnis des Typs für die Du rchführung der immunologischen Methode Voraussetzung. Beim Peroxydaseverfahren spielt dagegen der genetische Typ nur bei der Bewertung der Ergebnisse eine Rolle. Da die Normwertbereiche der Hämoglobinbindungskapazität der 3 genetischen Typen aber nur gering differieren, ist die Typendifferenzierung bei der Peroxydasemethode für praktische Belange von untergeordneter Bedeutung.

\section{The quantitative determination of serum baptoglobin by the peroxidase activation metbod and by radial immunodiffusion. A comparison of methods}

The peroxidase activation method of JAYLE and the radial immunodiffusion method according to MANCINI and coworker for the quantitative determination of serum haptoglobin were compared. The methodical errors of both methods were of about the same magnitude. The statistical comparison of the values obtained by each method showed significant correlations for the 3 genetic haptoglobin types and a characteristic regression line for each type.

Higher serum values were recorded with the immunological method than with the peroxidase method. This observation was due to differences in the reference systems between the protein concentration determined immunologically and the haemoglobin binding capacity determined by the peroxidase method. It is assumed that additional type-dependent differences were caused by different degrees of haemoglobin binding by the monomeric and polymeric forms. The appropriate regression equation is reported for the conversion of the immunologically determined haptoglobin concentrations into haemoglobin binding capacity (and vice versa).

When haemoglobin was added to the serum, the peroxidase method gave haptoglobin values that were too low, while the immunological method gave values that were too high. Different values for the normal ranges of the 3 genetic haptoglobin types are obtained by the two methods. Owing to the different behaviour of the 3 genetic haptoglobin types in the radial immunodiffusion, the type must first be known before the immunological method is performed. In the peroxidase method, however, the genetic type need only be considered in the evaluation of the results. Since the normal ranges of haemoglobin binding capacity of the three genetic types differ only very slightly, the type differentiation by the peroxidase method is, for practical purposes, of minor importance.

Das $\alpha_{2}$-Glykoprotein Haptoglobin bindet Hämoglobin unter Bildung eines Haptoglobin-Hämoglobin-Komplexes. Gelangt freies Hämoglobin in die Zirkulation, erfolgt ein rascher Transport des gebildeten Komplexes aus dem Serum in das retikuloendotheliale System. Bei allen Formen der Hämolyse finden sich daher erniedrigte Konzentrationen von freiem Haptoglobin im Serum (1). Da Haptoglobin in der Leberzelle gebildet wird (2), können auch Leberparenchymschäden auf dem Weg der Synthesestörung $z u$ einer Hypohaptoglobinämie führen (3, 4, 5). Verschiedene unspezifische Gewebsreaktionen beantwortet der Or- ganismus mit einer gesteigerten Haptoglobinsynthese, so daß es zu einer Erhöhung der Serumkonzentration kommt (6). Die quantitative Haptoglobinbestimmung im Serum besitzt demnach für alle K̇rankheitsprozesse Bedeutung, die mit herabgesetzter bzw. gesteigerter Synthese oder mit erhöhtem Verbrauch dieses Proteins einhergehen.

Für die quantitative Haptoglobinbestimmung stehen chemische, elektrophoretische, immunologische und Gelfiltrationsverfahren zur Verfügung (s. bei 7). Die Peroxydaseaktivierungsmethode nach JAYLE (8) eignet sich wegen ihrer raschen Durchführbarkeit und Zu- 
verlässigkeit vorwiegend für klinische Laboratorien mit hoher Untersuchungsfrequenz. Sie beruht auf der starken Äthylhydroperoxydaseaktivität des Haptoglobin-Hämoglobin-Komplexes gegenüber der ungleich geringeren Aktivität des ungebundenen Hämoglobins. Wird eine bestimmte Serummenge mit Hämoglobin versetzt, so katalysiert der gebildete HaptoglobinHämoglobin-Komplex folgende Reaktion:

$\mathrm{C}_{2} \mathrm{H}_{5} \mathrm{OOH}+2 \mathrm{~J}^{-}+2 \mathrm{H}^{+} \longrightarrow \mathrm{C}_{2} \mathrm{H}_{5} \mathrm{OH}+\mathrm{J}_{2}+\mathrm{H}_{2} \mathrm{O}$

Das freigesetzte Jod kann mit Stärke als Indikator gegen Natriumthiosulfat titriert werden:

$$
\mathrm{J}_{2}+2 \mathrm{Na}_{2} \mathrm{~S}_{2} \mathrm{O}_{3} \longrightarrow 2 \mathrm{NaJ}+\mathrm{Na}_{2} \mathrm{~S}_{4} \mathrm{O}_{6} \text {. }
$$

Das Volumen der verbrauchten Thiosulfatlösung ist direkt proportional der Haptoglobinkonzentration.

Wegen des mit dieser Methode verbundenen nicht unerheblichen technischen Aufwandes lag es nahe, einfacher zu handhabende Verfahren auf ihre Brauchbarkeit für die Haptoglobinbestimmung zu prüfen. Dafür schien die radiale Immunodiffusion nach MANCINI und Mitarbeitern (9) besonders geeignet.

In früheren Mitteilungen wurde bereits auf die Differenzen der mit beiden Methoden gewonnenen Haptoglobinwerte hingewiesen $(10,11,12)$. Diese sind zum Teil dadurch bedingt, da 3 verschiedene genetische Haptoglobintypen mit unterschiedlicher Primärstruktur existieren ( $\mathrm{Hp} \mathrm{1-1,} \mathrm{Hp} \mathrm{2-1,} \mathrm{Hp} \mathrm{2-2).} \mathrm{In} \mathrm{der} \mathrm{vor-}$ liegenden Studie sollen aufgrund eines Vergleiches zwischen der Peroxydasemethode und der radialen Immunodiffusion Gemeinsamkeiten, Unterschiede, Aussagekraft und Anwendbarkeit beider Methoden an einem größeren Untersuchungsgut geprüft werden.

\section{Methodik}

Quantitative Serumbaptoglobinbestimmung mit der Peroxydaseaktivierungsmethode nach JAYLE (8) in der von NYMAN (13) angegebenen Modifikation über Messung der Hämoglobinbindungskapazität (das ist die Menge Hämoglobin in $g$, die von dem in 11 Serum enthaltenen Haptoglobin gebunden wird (14)). Doppelbestimmungen und Angabe des Mittelwertes.

Quantitative Serumbaptoglobinbestimmung mit der einfachen radialen Immunodiffusion nach MANCINI und Mitarbeitern (9) in der Modifikation von BECKER und Mitarbeitern (15) auf Partigen-Platten der Behring-Werke, Marburg, mit Antiserum von Kaninchen gegen ein Antigengemisch der 3 menschlichen Haptoglobintypen $\mathrm{Hp} 1-1, \mathrm{Hp}_{\mathrm{p}} 2-1$ und $\mathrm{Hp}$ 2-2. Aufstellung der Eichkurven anhand von 4 verschiedenen Verdünnungen

a) eines Standardserums der Behring-Werke, Marburg, mit einem Gemisch der 3 genetischen Haptoglobintypen und bekanntem Gesamthaptoglobingehalt b) von in dest. Wasscr gelösten Rcinproteinen der 3 genetischen Haptoglobintypen (Ermittlung der Konzentration über Einwaage und spezifische Extinktion $\mathrm{E}_{280} \mathrm{~nm}$ : $\mathrm{Hp} 1-1=12,5 ; \mathrm{Hp} 2-1=$ 12,8; Hp $2-2=13,5)$. Doppelbestimmungen in 2 verschiedenen Verdiunnungsstufen und Angabe des Mittelwertes.

Differenzierung der genetiscben Haptoglobint'pen mit der vertikalen Stärkegelelektrophorese nach SMIrHIES (16) und anschließender Benzidinfärbung.

\section{Untersucbungsgut und methodisches Vorgeben}

1. Haptoglobintypendifferenzierung an insgesamt 373 Seren gesunder Erwachsener, Blutspender und Patienten mit vcrschiedenen inneren Erkrankungen.

2. Bestimmung des methodischen Fehlers (Variationskoeffizient V) unter verschiedenen Versuchsbedingungen für Peroxydascmethode und radiale Immunodiffusion.

a) 8fach-Bestimmung eines Serums in einem Versuchsansatz

b) Bestimmung cines in 10 Portionen abgefüllten Serums in 10 verschicdenen Versuchsansätzen.

3. Prüfung des Einflusses der Serumkonservierung auf die mit der Peroxydasemethode und mit der radialen Immunodiffusion gewonnenen Ergebnissc: Bestimmung der Serumhaptoglobinkonzentration vor und nach

a) Tiefkühlung $\left(-20^{\circ}\right)$ über länger als $1 \mathrm{Jahr}$

b) 12 maligem Einfrieren und Auftauen;

c) 3-4 wöchiger Tiefkühlung.

4. Bestimmung der Hämoglobinbindungskapazität mit der Peroxydasemethode und der Haptoglobinkonzentration mit der radialen Immunodiffusion in 107 Seren (unter Verwendung eines Standardmischserums zur Aufstellung der Eichkurven).

5. Bestimmung der Hämoglobinbindungskapazität mit der Peroxydasemethode und der Haptoglobinkonzentration mit der radialen Immunodiffusion in 266 Seren (unter Verwendung von Reinproteinen zur Aufstellung der Eichkurven). Ermittlung typenspezifischer Wcrte durch Ablesung über die jedem Haptoglobintyp entsprechende Reinprotein-Eichkurve.

6. Bestimmung der Haptoglobinkonzentration mit der radialen Immunodiffusion wie unter 5. nach Sättigung von 165 Seren mit Hämoglobin entsprechend der Hämoglobinbindungskapazität.

7. Aufstellung von Normwertbereichen für die mit beiden Methoden gewonnenen Werte nach genetischen Typen getrennt.

8. Statistische Methoden: Ermittlung der Regressionsgeraden für die mit der Peroxydasemethode und der radialen Immunodiffusion gewonnenen Wertepaare. Für die verschiedenen Vergleiche der Stichproben und Mittelwerte Anwendung von parameterfreien Testmethoden (Friedman-, Wilcoxon-, KRUSKal-Wallis-; MANN-WhrtNEY-Test). Signifikanzniveau für alle. Methoden, wenn nicht anders angegeben (Tab. 2) $2 \alpha=0,05$.

\section{Ergebnisse}

Für die Peroxydasemethode und die radiale Immunodiffusion wurde ein gleich großer methodischer Fehler $(V=3,9)$ ermittelt, wenn Mehrfachbestimmungen eines Serums in einem Versuchsansatz durchgeführt wurden (Tab. 1). Erfolgten dagegen die Unter-

Tab. 1

Bestimmung des methodischen Fehlers (=Variationskoeffizient V) unter verschiedenen Versuchsbedingungen für Peroxydasemethode und radiale Immunodiffusion. a) 8 fach-Bestimmung eines Serums in einem Versuchsansatz, b) Bestimmung eines in 10 Portionen abgefüllten Serums in 10 verschiedenen Versuchsansätzen

\begin{tabular}{|c|c|c|c|c|}
\hline $\begin{array}{c}\text { Mẹthode } \\
\text { Versuchsbedingungen }\end{array}$ & $\begin{array}{c}\text { Peroxydasemethode } \\
\text { a }\end{array}$ & $\underset{a}{\substack{\text { Radiale } \\
\text { Immunodiffusion }}}$ & $\begin{array}{c}\text { Peroxydasemethode } \\
\text { b }\end{array}$ & $\underset{\substack{\text { Radiale } \\
\text { I mmunodiffusion }}}{x}$ \\
\hline $\begin{array}{l}\overline{\mathbf{x}}[\mathrm{g} / 1] \\
\mathrm{s}[\mathrm{g} / 1] \\
\mathrm{n}\end{array}$ & $\begin{array}{l}0,85 \\
0,033 \\
8\end{array}$ & $\begin{array}{l}4,49 \\
0,174 \\
8\end{array}$ & $\begin{array}{l}1,94 \\
0,140 \\
10\end{array}$ & $\begin{array}{l}3,80 \\
0,414 \\
10\end{array}$ \\
\hline . $\quad V=\frac{s}{\bar{x}} \cdot 100$ & 3,9 & 3,9 & 7,2 & 10,9 \\
\hline
\end{tabular}


Tab. 2

Einfluß der Lagerung auf die Serumhaptoglobinkonzentration; Temperatur bei Tiefkühlung $-20^{\circ}$, Signifikanzniveau $2 \alpha=0,10$

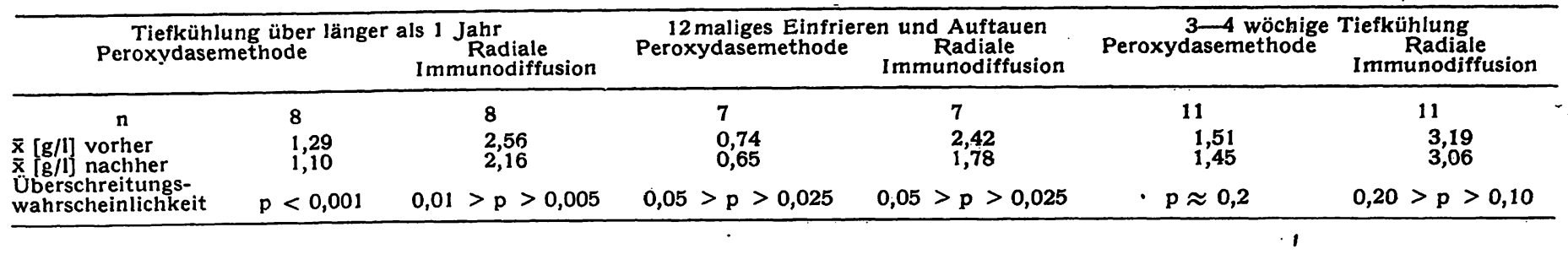

Tab. 3

Vergleich der Haptoglobinmittelwerte: Peroxydasemethode — radiale Immunodiffusion vor und nach Hämoglobinzusatz (keine Differenzierung in normale und pathologische Fälle)

\begin{tabular}{|c|c|c|c|c|}
\hline Genetischer Typ & $\begin{array}{l}\text { Peroxydase-Methode } \\
\text { g/l Hämoglobin- } \\
\text { bindungskapazität }\end{array}$ & $\begin{array}{l}\text { Radiale Immuno } \\
\text { vor Hämoglobin- } \\
\text { sättigung }\end{array}$ & $\begin{array}{c}\text { ffusion g/l Protein } \\
\text { nach Hämoglobin- } \\
\text { sättigung }\end{array}$ & $\begin{array}{l}\text { Unterschiede bei } \\
\text { paarweisem Vergleich } \\
\text { der Mittelwerte }\end{array}$ \\
\hline Hp 1-1 & $\begin{aligned} \mathrm{n} & =45 \\
\overline{\mathrm{x}} & =1,84 \\
\mathrm{~s} & =1,20\end{aligned}$ & $\begin{aligned} n & =45 \\
\bar{x} & =2,75 \\
s & =1,72\end{aligned}$ & $\begin{array}{l}n \cdot=45 \\
\bar{x} \doteq 2,95 \\
s=1,84\end{array}$ & $p<0,00001$ \\
\hline Hp 2-1 & $\begin{array}{l}n=50 \\
\bar{x}=1,84 \\
s=1,55\end{array}$ & $\begin{array}{l}\mathrm{n}=50 \\
\bar{x}=3,91 \\
\mathrm{~s}=3,32\end{array}$ & $\begin{aligned} \mathrm{n} & =50 \\
\overline{\mathrm{x}} & =4,47 \\
\mathrm{~s} & =3,89\end{aligned}$ & $\dot{p}<0,00001$ \\
\hline Hр 2-2 & $\begin{array}{l}\mathrm{n}=70 \\
\overline{\mathrm{x}}=1,36 \\
\mathrm{~s}=1,47\end{array}$ & $\begin{aligned} \mathrm{n} & =70 \\
\overline{\mathrm{x}} & =3,15 \\
\mathrm{~s} & =3,27\end{aligned}$ & $\begin{array}{l}\mathrm{n}=70 \\
\overline{\bar{x}}=3,22 \\
\mathrm{~s}=3,26\end{array}$ & $p=0,02$ \\
\hline
\end{tabular}

Abb. 1

Wertepaare und Regressionsgeraden für immunologisch $(x=g / 1$ Haptoglobin) und mit der Peroxydasemethode ( $y=g / 1$ Hämoglobinbindungskapazität) bestimmte $\mathrm{Se}$ rumhaptoglobinkonzentrationen der 3 genetischen Haptoglobintypen. Eichkurven für die radiale Immunodiffusion aus der Verwendung von Standardserum gewonnen.

- Haptoglobin $1-1 \mathrm{y}=0,24+0,49 x$; $\mathrm{r}=0,95 ; \mathrm{n}=36$

+ Haptoglobin 2-1 y $=0,11+0,65 x$;

ㅁ Haptoglobin $2-2 \mathrm{y}=0,11+0,76 \mathrm{x}$;

$\mathrm{r}=0,97 ; \mathrm{n}=31$

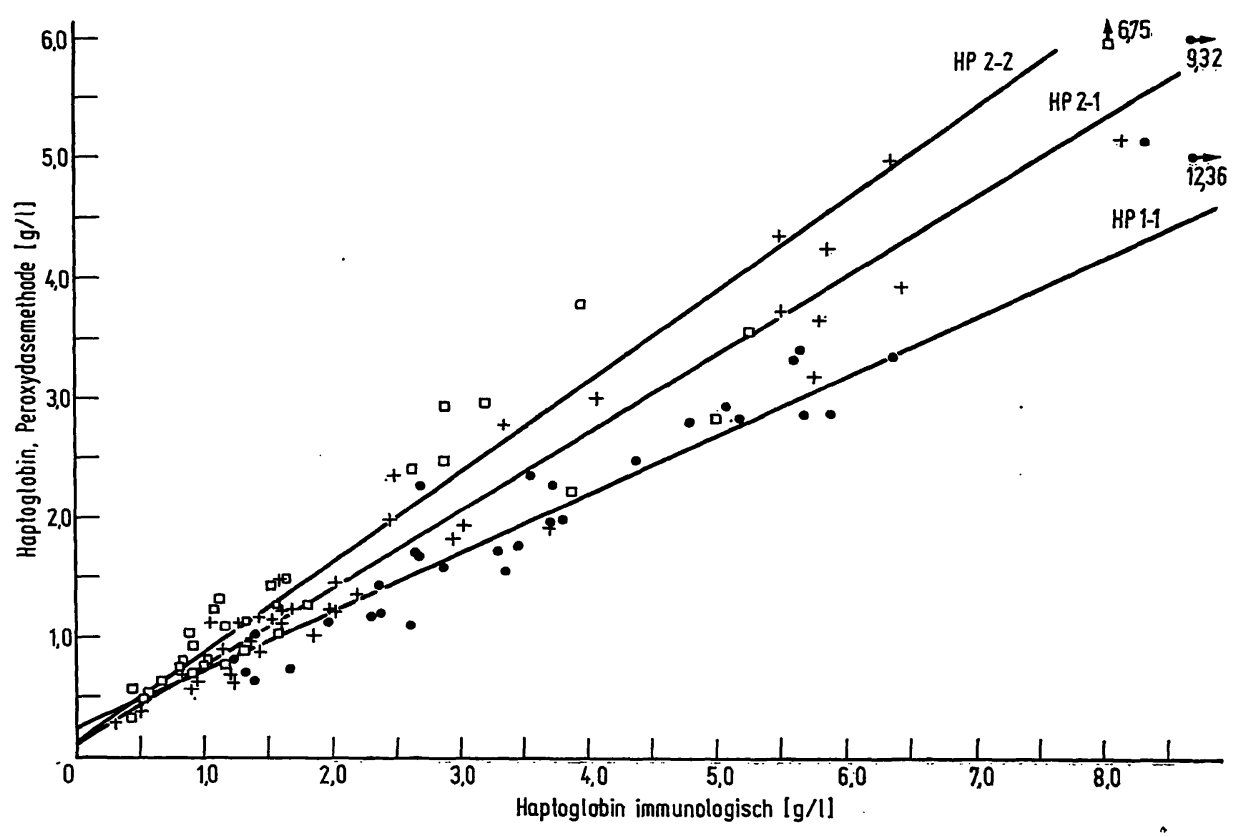

suchungen in verschiedenen Ansätzen, erwies sich der Fehler der immunologischen Methode gegenüber dem des Peroxydaseverfahrens als etwas größer (Tab. 1). Der Einfluß der Serumkonservierung auf die Haptoglobinwerte beider Methoden geht aus Tabelle 2 hervor. Eine gleichsinnige Abnahme der Hämoglobinbindungskapazität und der immunologisch bestimmten Werte trat ein, wenn die Seren länger als $1 \mathrm{Jahr}$ bei $-20^{\circ}$ lagerten, oder wenn bei kürzerer Lagerungszeit oftmaliges Einfrieren und Auftauen erfolgten. 3-4 Wochen tiefgefroren gelagerte Seren zeigten dagegen keine signifikanten Konzentrationsänderungen.

Der Vergleich zwischen immunologisch und mit der Peroxydasemethode bestimmten Haptoglobinwerten ergab in jeder Versuchsanordnung signifikant höhere Werte bei Anwendung der radialen Immunodiffusion (Tab. 3). Wurde für die immunologische Methode zur
Herstellung der Eichkurven gepooltes Standardserum verwandt, fandèn sich die größten Ergebnisdifferenzen beim Haptoglobintyp $\mathrm{Hp} 1-1$, die kleinsten bei $\mathrm{Hp}$ $2-2$, dazwischen lagen die für den Typ Hp 2-1. Dem entsprach das kleinste Steigungsmaß der Regressions-

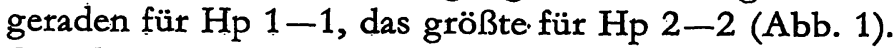
Für die mit beiden Methoden bestimmten Werte ergaben sich positive Korrelationen für alle 3 genetischen Typen (Abb. 1).

Bei Verwendung von Reinproteinen der 3 genetischen Haptoglobintypen zur Herstellung der Eichkurven für die immunologische Methode fanden sich andere Ergebnisse. Zwar wurden auch unter diesen Versuchsbedingungen hohe Korrelationskoeffizienten bestimmt (Abb. 2). Die geringste Steigung zeigte jetzt aber die Regressionsgerade des Typs $\mathrm{Hp} 2-2$, die stärkste die für Hp 1-1. Die Differenzen der mit beiden Methoden 


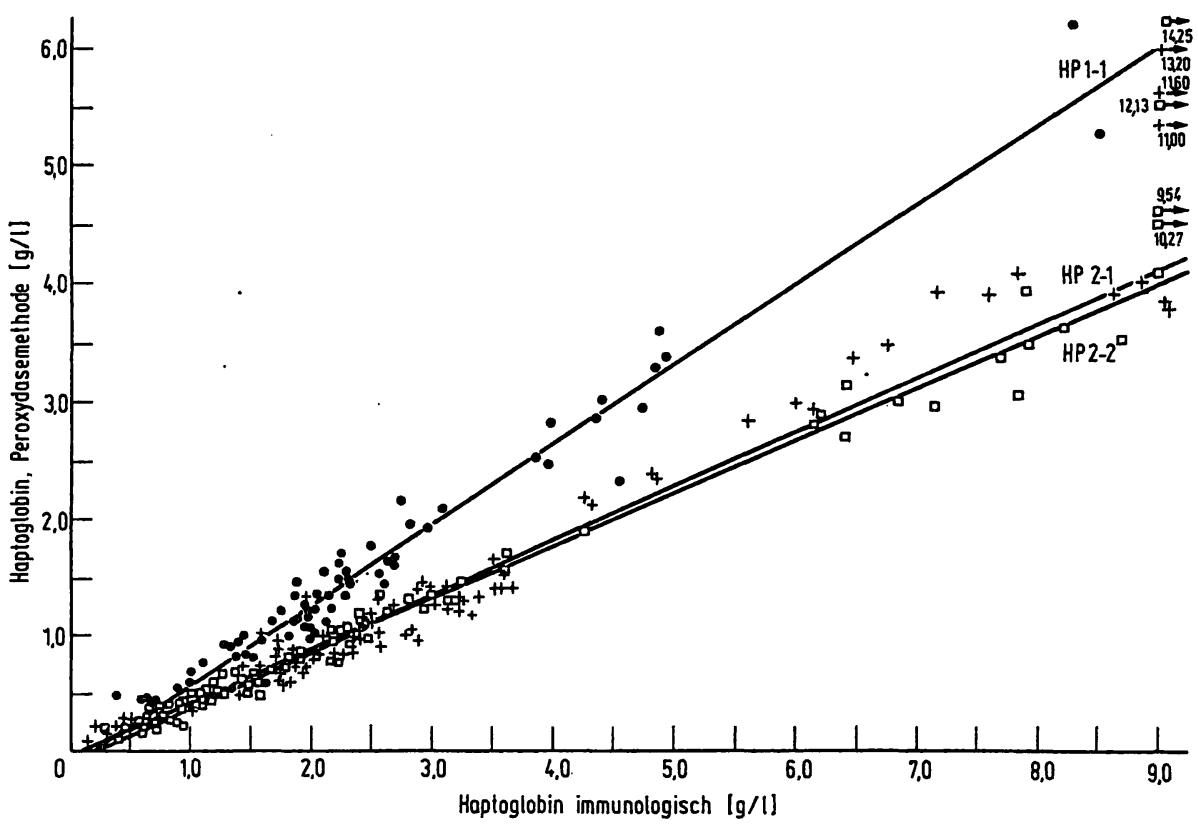

Abb. 2

Wertepaare und Regressionsgeraden für immunologisch ( $x=g / 1$ Haptoglobin) und mit der Peroxydasemethode ( $y=g / 1$ Hämoglobinbindungskapazität) bestimmte Serumhaptoglobinkonzentrationen der 3 genetischen Haptoglobintypen. Eichkurven für die radiale Immunodiffusion aus der Verwendung von Reinproteinen der 3 genetischen Haptoglobintypen gewonnen

- Haptoglobin $1-1$ y $y=-0,14+0,69 x$; $\mathrm{r}=0,97 ; \mathrm{n}=72$

$+\underset{r=0,99 ; n=96}{\text { Haptoglobin } 2-1} \mathrm{y}=-0,09+0,47 x$ $\square \underset{r=0,99 ; n=98}{\text { Haptoglobin } 2-2} \mathrm{y}=-0,04 \times 0,45 x$; gewonnenen Ergebnisse waren demnach für $\mathrm{Hp} 1-1$ kleiner als für die beiden anderen Typen. Der Quotient aus den immunologisch und mittels des Peroxydaseverfahrens bestimmten Werten (Proteinbindungsquotient des Haptoglobins) war für die 3 Haptoglobıntypen verschieden (Tab. 4): am größten für $\mathrm{Hp} \mathrm{2-2}$ und am kleinsten für $\mathrm{Hp} 1-1$.

Tab. 4

Proteinbindungsquotienten der $\mathbf{3}$ genetischen Haptoglobintypen

\begin{tabular}{ccc}
\hline Hp 1-1 & Hp 2-1 & Hp 2-2 \\
\hline $\mathrm{n}=72$ & $\mathrm{n}=96$ & $\mathrm{n}=98$ \\
$\overline{\mathrm{x}}=1,64$ & $\overline{\mathrm{x}}=2,34$ & $\overline{\mathrm{x}}=2,45$ \\
$\mathrm{~s}=0,32$ & $\mathrm{~s}=0,37$ & $\mathrm{~s}=0,32$ \\
\hline
\end{tabular}

Zur Deutung dieser auffälligen Differenzen wurde der Verlauf der mit Reinproteinen und Standardserum gewonnenen Eichkurven untersucht (Abb. 3). Dabei ergab sich. für die Kurve des $\mathrm{Hp} 1-1$ die stärkste Steigung, die Eichkurve des Standardserums verlief

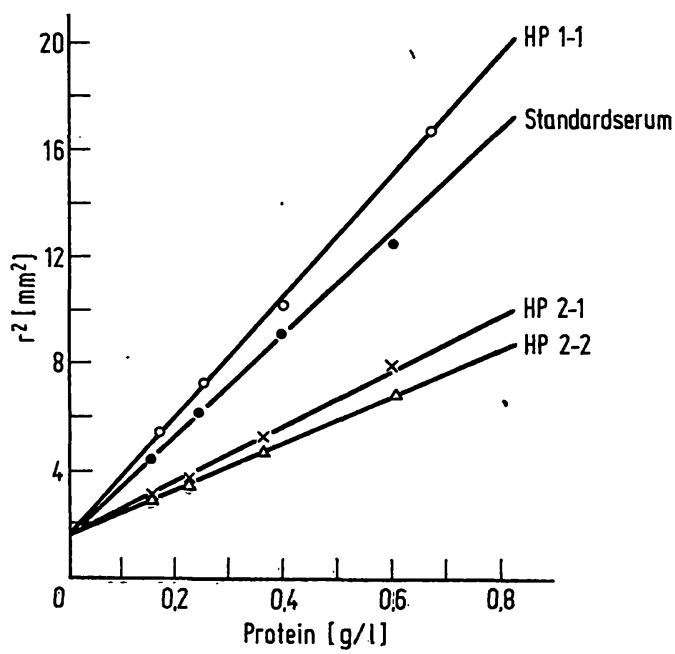

Abb. 3

Radiale Immunodiffusion. Eichkurven für die 3 Reinproteine der 3 genetischen Haptoglobintypen und ein Standardmischserum $(x=g / 1$ Haptoglobin; $y=r^{2}$ der Immunpräzipitate) zwischen der für Hp 2-1 und $\mathrm{Hp} 1-1$. Das bedeutet, daß die Präzipitatdurchmesser des Typs $\mathrm{Hp} 1-1$ für die gleichen Proteinkonzentrationen größer waren als die der beiden anderen genetischen Typen.

Nach Sättigung der Seren mit Hämoglobin entsprechend der jeweiligen Hämoglobinbindungskapazität nahmen die immunologisch bestimmten Haptoglobinkonzentrationen $\mathrm{zu}$ (Tab. 3). Nach dem Wilcoxon-Test waren die Unterschiede der Mittelwerte für alle Typen signifikant.

Mit beiden Bestimmungsverfahren konnten verschiedene Normwertbereiche aufgestellt werden (Tab. 5). Unterschiede ergaben sich aber nicht nur hinsichtlich der beiden Methoden im Gesamtkollektiv, sondern

Tab. 5

Mit der Peroxydasemethode und der radialen Immunodiffusion ermittelte Normwertbereiche für die 3 genetischen Haptoglobintypen

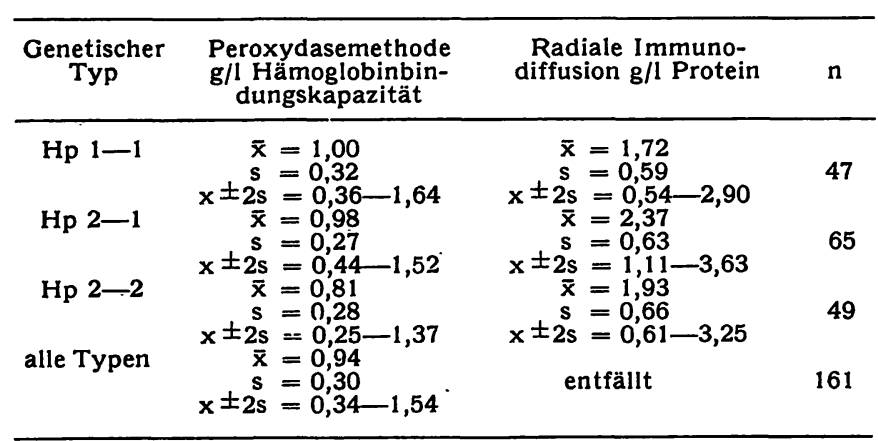

auch für die 3 genetischen Typen unabhängig von der angewandten Methode. Dabei waren die Differenzen zwischen den mittels Peroxydaseverfahren bestimmten Werten der Typen Hp $1-1$ und Hp 2-2 (p < 0,005) sowie zwischen $\mathrm{Hp} 2-1$ und $\mathrm{Hp} 2-2$ ( $2<0,005)$ signifikant, während die Unterschiede bei den Typen $\mathrm{Hp} 1-1$ und $\mathrm{Hp} 2-1(\mathrm{p} \approx 0,56)$ zufällig waren.

Für die immunologische Bestimmung fanden sich signifikante Differenzen zwischen $\mathrm{Hp} 1-1$ und $\mathrm{Hp} 2-1$ $(\mathrm{p}<0,00001)$ sowie $z$ wischen $\mathrm{Hp} 2-1$ und $\mathrm{Hp} 2-2$ 
$(\mathrm{p}<0,001)$, dagegen nicht zwischen $\mathrm{Hp} 1-1$ und Hp 2-2 $(p \approx 0,33)$. Die niedrigsten mit der Peroxydasemethode gemessenen Normalwerte zeigte $\mathrm{Hp}$ $2-2$ (Tab. 5), für die radiale Immunodiffusion $\mathrm{Hp} 1-1$.

\section{Diskussion}

\section{Metbodischer Febler und Bedeutung der Serumkonservierung}

Die beiden untersuchten Haptoglobinbestimmungsverfahren weisen einen gleich großen methodischen Fehler auf (Tab. 1) (10), der unter bestimmten Bedingungen für die radiale Immunodiffusion vergleichsweise etwas höher ist. Beide Methoden erfüllen die Kriterien einer ausreichenden Reproduzierbarkeit. Mit dem Peroxydaseverfahren können die Ergebnisse innerhalb weniger Minuten erstellt werden, die radiale Immunodiffusion erfordert eine Diffusionszeit von 36-48 Stunden.

Der Einfluß der Serumkonservierung auf die Ergebnisse der Haptoglobinbestimmung wird in der Literatur verschieden beurteilt (13, 17). Während Nyman (13) Änderungen der Hämoglobinbindungskapazität weder bei Aufbewahrung über länger als 1 Jahr bei $-15^{\circ}$ noch bei wiederholtem Einfrieren und Auftauen beobachten konnte, beschrieben COHNEN und PAAR (17) einen mit der Immunodiffusion ermittelten Konzentrationsverlust sowohl nach 20 monatiger Lagerung bei $-28^{\circ}$ als auch bereits nach 4 maligem Einfrieren und Auftauen. Unsere Untersuchungen bestätigten die Ergebnisse der letztgenannten Autoren (Tab. 2). Von Bedeutung erscheint jedoch die Beobachtung, daß der Abfall der Werte für beide Methoden ungefähr das gleiche Ausmaß hatte, und daß während 3-4 Wochen bei $-20^{\circ}$ gelagerte Seren keine signifikanten Meßwertänderungen zeigten.

\section{Vergleich der Mitteliverte beider Metboden}

Beim Vergleich der mit der Peroxydasemethode und mit der radialen Immunodiffusion gewonnenen Haptoglobinwerte fällt auf, daß die immunologisch bestimmten Mittelwerte für die 3 genetischen Haptoglobintypen signifikant höher liegen (Tab. 3). Diese Differenzen erklären sich zum Teil daher, daß für beide Methoden unterschiedliche Bezugssysteme zur Anwendung kommen. Zwar werden die Ergebnisse beider Verfahren in der gleichen Meßeinheit ( $\mathrm{g} /$ Volumen) angegeben. Während die Peroxydasemethode jedoch nur indirekt über die Hämoglobinbindungskapazität eines Serumvolumens auf den Haptoglobingehalt schließen läßt, erlaubt die radiale Immunodiffusion eine Bestimmung der Proteinkonzentration. Da Haptoglobin Hämoglobin (bei Hämoglobinüberschuß) in äquimolarem Verhältnis bindet (18) und die 3 Haptoglobintypen höhere Molekulargewichte als Hämoglobin besitzen (Úbersicht bei 7), kann pro Gewichtseinheit Haptoglobin nicht der gleiche Gewichtsanteil Hämoglobin gebunden werden. Daher müssen die immunologisch bestimmten Werte für die 3 Typen über denen der Hämoglobinbindungskapazität liegen.

\section{Typenspezifiscbe Wertdifferenzen}

Berücksichtigt man die heutigen Vorstellungen über die unterschiedlichen Molekülgrößen der 3 genetischen Hp-Typen, so sind außerdem typenspezifische Differenzen $z$ wischen den immunologisch und den mit der Peroxydasemethode ermittelten Werten zu erwarten. Unter Zugrundelegung eines Molekulargewichtes von 100000 für $\mathrm{Hp} 1-1$ (19) und von 64500 für Hämoglobin (20), beträgt bei äquimolar'er Bindung das $\mathrm{Ge}-$ wichtsverhältnis von Haptoglobin zu Hämoglobin $\frac{100000}{64500}=1,55$. Dieser Wert entspricht also auch dem Verhältnis zwischen immunologisch bestimmter Proteinkonzentration und der Hämoglobinbindungskapazität („Proteinbindungsquotient des Haptoglobins" (11)). Die früher angegebenen (11), von den vorliegenden Ergebnissen (Tab. 4) etwas abweichenden Proteinbindungsquotienten dürften mit der geringeren Anzahl der untersuchten Seren zusammenhängen. Aus Tabelle 4 ergibt sich für $\mathrm{Hp} 1-1$ eine relativ gute Übereinstimmung zwischen gefundenem und errechnetem (s. o.) Proteinbindungsquotienten.

Komplizierter liegen die Verhältnisse für $\mathrm{Hp} 2-1$ und Hp 2-2, da bisher infolge der ausgeprägten Neigung beider Typen zur Polymerisation weder zuverlässige Angaben über die Molekulargewichte noch über das quantitative Hämoglobinbindungsvermögen vorliegen. Als weitgehend gesichert kann aber gelten, daß die Molekulargewichte der Typen Hp 2-1 und Hp 2-2 im Vergleich zum Hp 1-1-Molekül deutlich höher liegen (s. bei 7). Dafür sprechen vor allem neuere Untersuchungen über die Primärstruktur der Polypeptidketten der kleinsten Haptoglobinmoleküleinheiten $(21,22)$.

Aus den vergleichsweise höheren Proteinbindungsquotienten für $\mathrm{Hp} 2-1$ und $\mathrm{Hp} \mathrm{2-2}$ (Tab. 4) kann jedoch nicht ohne weiteres auf die Höhe der Molekulargewichte geschlossen werden. Nimmt man an, daß die Monomeren der Typen $\mathrm{Hp} 2-1$ und $\mathrm{Hp} \mathrm{2-2}$ Hämoglobin äquimolar zu binden vermögen (19), so lassen sich unter Berücksichtigung der Molekulargewichte der Hp-Untereinheiten (21, 22, 23) niedrigere Proteinbindungsquotienten berechnen, als sie in den eigenen Untersuchungen ermittelt wurden. Die Beobachtung höherer Proteinbindungsquotienten für die Typen Hp 2-1 und Hp 2-2 (Tab. 4) läßt demnach die Annahme zu, daß die polymerisierten Typen weniger Hämoglobin binden als die monomere Form Hp 1-1. Diese Hypothese steht im Gegensatz zu der von CroARE'c und Moretti (24) und von Hamaguchr. (25) vertretenen Ansicht, nach der das Hämoglobinbindungsverhältriis bei den 3 genetischen Typen gleich ist. Andererseits geht aus den Untersuchungen von JAvid (26) hervor, daß die höher polymerisierten Fraktionen von $\mathrm{Hp} 2-1$ und $\mathrm{Hp} 2-2$ pro Gewichtseinheit weniger Hämoglobin binden als die niedrigen. Es ist also denkbar, daß bei gleichem molarem Hämoglobinbindungsverhältnis der Untereinheiten in den höheren Polymeren durch Änderungen der Sekundär- und Tertiärstruktur 
cine sterische Behinderung der Hämoglobinbindung eintritt, aus der eine im Hinblick auf das Gesamtmolekül niedrigere Hämoglobinbindungskapazität resultiert $(26,27)$. Eine ähnliche Deutung könnten aụch die Beobachtungen von Ogawa und Kawamura (28) zulassen. Ein Einfluß antigenischer Verschiedenheiten der 3 genetischen Haptoglobintypen auf die Versuchsergebnisse konnte dagegen nicht wahrscheinlich gcmacht werden $(7,11)$.

\section{Einfluß der Eichproteine auf die radiale Immunodiffusion}

Der Nachweis typenspezifischer Regressionsgeraden bei der Korrelation immunologisch und mit der Peroxydasemethode bestimmter Haptoglobinwerte (Abb. $1,2)$ ist durch die diskutierten Zusammenhänge erklärt. Der differente Verlauf der 3 Geraden bei Verwendung verschiedener Eichproteine für die radiale Immunodiffusion hat andere Ursachen. Aus Abbildung 3 wird deutlich, daß sich unter Zugrundelegung von gepooltem Standardserum und von Reinproteinen der 3 genetischen Haptoglobintypen jeweils verschiedene Eichkurven ergeben. Die Präzipitatdurchmesser gleicher Proteinkonzentrationen sind demnach am größten für $\mathrm{Hp} 1-1$, am kleinsten für $\mathrm{Hp} 2-2$, während die für Hp 2-1 und das verwandte Standardserum eine Mittelstellung einnehmen. Am Zustandekommen dieser Differenzen könnten unterschiedliche Diffusionskoeffizienten der 3 Haptoglobintypen beteiligt sein.

Sie lassen sich aber einfacher über die verschiedenen Molekulargewichte der 3 Haptoglobintypen deuten. Danach erscheint einleuchtend, daß pro Gewichtseinheit des Haptoglobintyps mit dem kleinsten Molekulargewicht ( $\mathrm{Hp} \mathrm{1-1)}$ mehr Moleküle mit dem korrespondierenden Antiserum reagieren können, als im Falle der beiden schwereren Proteine. Die Kurve des Typs Hp 1-1 muß daher die stärkste Steigung aufweisen. Der Kurvenverlauf bei Verwendung von Standardserum mit einem Gemisch aller 3 Typen ist abhängig vom mengenmäßigen Anteil eines jeden enthaltenen Proteintyps. Werden infolgedessen unter den beschriebenen Versuchsbedingungen (Abb. 3) Haptoglobinkonzentrationen eines Serums vom Typ Hp 1-1. über die Eichkurve aus Standardserum ermittelt, so müssen die gewonnenen Werte $\mathrm{zu}$ hoch liegen; in Seren der Typen $\mathrm{Hp} 2-1$ und $\mathrm{Hp} 2-2$ werden dagegen $\mathrm{zu}$ niedrige Konzentrationen bestimmt (12, 29). Die in Abbildung 1 dargestellten Regressionsgeraden beziehen sich auf Standardserum als Eichantigen für die radiale Immunodiffusion und müssen sich deshalb von den in Abbildung 2 dargestellten Untersuchungsergebnissen unterscheiden. Die früher beschriebenen andersartigen Proteinbindungsquotienten (10) beziehen sich ebenfalls auf Standardserum als Eichproteinlösung.

Voraussetzung für eine exakte quantitative Haptoglobinbestimmung mit Hilfe der radialen Immunodiffusion ist demnach die Kenntnis des genetischen Haptoglobintyps. Darüber hinaus müssen die Eichkurven aus den entsprechenden Reinproteinen der
3 Haptoglobintypen erstellt werden. Wird dagegen ein Standardmischserum zur Eichung verwandt, sind für jede Charge neu zu erstellende Umrechnungsfaktoren zu berücksichtigen.

\section{Empfindlicbleit beider Metboden bei niedrigen Haproglobir- konzentrationen}

Für die Erfassung niedrigster Haptoglobinkonzentrationen im Serum ist die radiale Immunodiffusion die verläßlichere Methode. Nach Lage der Schnittpunkte der 3 Regressionsgeraden mit der Abszisse (Abb. 2) ist zu erwarten, daß immunologisch noch Proteinkonzentrationen bestimmt werden können, wenn die Hämoglobinbindungskapazität auf nicht mehr meßbare Werte erniedrigt ist. Diesc Vermutung ließ sich experimentell bestätigen: in sämtlichen Seren ohne meßbare Hämoglobinbindungskapazität konnte mit der radialen Immunodiffusion noch Haptoglobin nachgewiesen werden. Exakte Konzentrationsangaben sind dabei allerdings nicht möglich, da eine Typendifferenzierung bei fehlender Peroxydascaktivität nicht gelingt. Der Befund einer nicht meßbaren Peroxydaseaktivität berechtigt daher noch nicht zur Diagnose Anhaptoglobinämie $(30,31)$.

\section{Einfluß der Hämolyse auf beide Methoden}

Die Auswirkung hämolytischer Seren auf die Hämoglobinbindungskapazität ist von NYMAN (13) eingehend untersucht worden. Für die Durchführung der Peroxydasemethode hat sich die Verwendung von Pferdehämoglobin als «weckmäßig erwiesen, nachdem JAYLE (18) bereits 1940 festgestcllt hatte, daß die Peroxydaseaktivität des Haptoglobinkomplexes mit Pferdehämoglobin die des Komplexes mit menschlichem Hämoglobin weit übertrifft. Für die Berechnung der Hämoglobinbindungskapazität aus dem Volumen des titrierten Thiosulfats (s. Methodik) ergibt sich daher bei Verwendung von menschlichem Hämoglobin ein anderer Faktor als für Pferdehämoglobin (13). Wird die Peroxydasemethode, wie allgemein üblich, mit Pferdehämoglobin durchgeführt, müssen Seren, die menschliche Hämoglobinbeimengungen enthalten, zu niedrige Haptoglobinwerte liefern.

Die mit der radialen Immunodiffusion bestimmten Haptoglobinwerte liegen demgegenüber für hämoglobin-gesättigte Seren höher als für die gleichen Seren ohne Hämoglobinzusatz (Tab. 3). Während in einer früheren Untersuchung (11) die Differenzen zwischen den Serumkonzentrationen des Haptoglobintyps $\mathrm{Hp}$ 2-2 und seiner Komplexe mit Hämoglobin zufällig waren, ließ sich in der vorlicgenden Studie an einem größeren Untersuchungsgut die Signifikanz der Differenzen für alle 3 genetischen Typen sichern (Tab. 3). Dic Deutung dieses Phänomens ist schwierig. Von verschiedenen Untersuchern wurde eine Blockierung von Antigendeterminanten des Haptoglobins durch die Hämoglobinbindung nachgewiesen $(32-36)$. Für das den eigenen Untersuchungen zugrunde liegende System 
konnte jedoch eine wesentliche Bedeutung derartiger Vorgänge nicht wahrscheinlich gemacht werden (7). Es muß also dahingestellt bleiben, ob die Größenzunahme der Präzipitate ein einfaches quantitatives Phänomen im Sinne einer stärkeren räumlichen Ausdehnung der präzipitierten Antigen-Antikörperkomplexe durch Bindung zusätzlicher Hämoglobinmoleküle darstellt, oder ob kompliziertere Änderungen der Sekundär- oder Tertiärstruktur der beteiligten Moleküle sich auf den Präzipitationsmechanismus auswirken.

\section{Methoden- und typenspezifische Normivertbereiche}

Alirson und ReEs (37) haben erstmals darauf hingewiesen, daß sich für die genetischen Haptoglobintypen unterschiedliche Normwertbereiche aufstellen lassen. Ähnliche Befunde wurden in der Folge auch von anderen Untersuchern erhoben (Tab. 6). Darüber hinaus wird aufgrund der oben dargelegten Zusammenhänge verständlich, daß alle Verfahren, die auf der Messung der Hämoglobinbindungskapazität beruhen, andere Normwerte ergeben als die immunologischen Bestimmungsmethoden. Wie die Literaturauswahl (Tab. 6) erkennen läßt, stehen unsere mit der Peroxydasemethode gewonnenen Normalwerte weitgehend in Einklang mit den Angaben der meisten Autoren. Nur für den Haptoglobintyp $\mathrm{Hp} 1-1$ wurden von einigen Untersuchern deutlich höhere Werte ermittelt $(13,39$, 40, 42). Für die immunologische Methode liegen nur wenige vergleichbare Ergebnisse vor. Die von BECKER und Mitarbeitern (29) kürzlich mitgeteilten Normalwerte für die radiale Immunodiffusion entsprechen den von uns bestimmten fast vollständig. Auffallend niedrige Ergebnisse $(n=40, \bar{x}=129, s=42)$ erzielten GaBL und HUBER (45) mit einer Modifikation des Ouchterlony-Testes. Eine Typendifferenzierung erfolgte in dieser Studie nicht. Diskrepanzen finden sich im Hinblick auf die umfangreichen Untersuchungen von FauL (44). Der dort angestellte Vergleich zwischen der Peroxydasemethode und verschiedenen immunologischen Verfahren (Trübungsmessung, Nin- hydrinmethode, OuCHTERLONY-Test) ergab mit den angewandten statistischen Methoden keine signifikanten Normwertdifferenzen. Die insbesondere jedoch bei pathologischen Fällen häufig festgestellten höheren immunologischen Werte wurden als zufallsbedingt bzw. im Rahmen des methodischen Fehlers gedeutet.

\section{Konsequenzen für die Anwendbarkeit beider Metboden}

Für die quantitative Haptoglobinbestimmung eignen sich sowohl die Peroxydaseaktivierungsmethode als auch die radiale Immunodiffusion. Der methodische Fehler beider Verfahren ist etwa gleich groß und läßt auf eine ausreichende Reproduzierbarkeit schließen. Die Peroxydasemethode erfordert größeren technischen Aufwand und ist in der Handhabung zwar schneller, aber diffiziler als die radiale Immunodiffusion.

Die vorherige Bestimmung des genetischen Haptoglobintyps ist für die Durchführung der Peroxydasemethode nicht erforderlich. Demgegenüber ist die Kenntnis des Haptoglobintyps für die Ermittlung der Haptoglobinkonzentration mit der radialen Immunodiffusion unumgängliche Voraussetzung. Der geringere technische Aufwand der immunologischen Methode selbst wird daher durch die zusätzlich erforderliche Typendifferenzierung aufgewogen.

$\mathrm{Zu}$ berückssichtigen bleibt immer, daß für beide Methoden verschiedene Bezugssysteme gelten. Die für beide Verfahren ermittelten Normwertbereiche unterscheiden sich beträchtlich voneinander. Die Umrechnung der Ergebnisse der beiden Bestimmungsmethoden (g/1 Proteinkonzentration in Hämoglobinbindungskapazität bzw. umgekehrt) ist über die angegebenen Regressionsgleichungen (Abb. 2) unschwer möglich. Bei nicht meßbar niedriger Hämoglobinbindungskapazität läßt sich mit der radialen Immunodiffusion häufig noch Haptoglobin nachweisen. In diesen Fällen ist daher die immunologische Methode dem Peroxydaseverfahren überlegen. Ist dagegen die Hämoglobinbindungskapazität zwar erniedrigt, aber noch meßbar, besitzt die Peroxydasemethode die größere Aussagekraft, dà die

Tab. 6

Haptoglobinnormalwerte ( $g / 1)$ nach der Literatur (aufgeführt sind nur Angaben mit typendifferenzierten Werten in g/l)

\begin{tabular}{|c|c|c|c|c|c|c|c|c|c|c|c|c|c|}
\hline \multirow[b]{2}{*}{ Autoren } & \multirow[b]{2}{*}{ Methode } & \multicolumn{3}{|c|}{ Alle Typen } & \multicolumn{3}{|c|}{ Hp $1-1$} & \multicolumn{3}{|c|}{ Hp 2-1 } & \multicolumn{3}{|c|}{ Hp 2-2 } \\
\hline & & $\overline{\mathbf{x}}$ & & $\mathbf{n}$ & $\overline{\mathbf{x}}$ & s & $\mathbf{n}$ & $\overline{\mathbf{x}}$ & s & n & $\overline{\mathbf{x}}$ & & $\mathbf{n}$ \\
\hline NYMAN (13) & Peroxydase- & 1,10 & 0,41 & 277 & 1,36 & 0,30 & 59 & 1,08 & 0,37 & 130 & 0,82 & 0,34 & 86 \\
\hline SMITH, OWEN (27) & Peroxydase- & 0,93 & 0,40 & 152 & 1,04 & 0,34 & 21 & 1,02 & 0,37 & 80 & 0,72 & 0,38 & 49 \\
\hline BAYANI-SIOSON und Mitarbeiter (38) & Peroxydase- & 0,81 & 0,24 & 161 & 0,94 & 0,21 & 23 & 0,86 & 0,22 & 77 & 0,69 & 0,23 & 61 \\
\hline WHITTAKER (39) & Peroxydase- & 0,92 & 0,38 & 102 & 1,15 & 0,26 & 9 & 0,93 & 0,32 & 50 & 0,90 & 0,39 & 40 \\
\hline ALLISON $(40)$ & $\begin{array}{l}\text { Stärkegelelektropho- } \\
\text { rese }\end{array}$ & - & - & - & 1,20 & 0,26 & 一 & 1,20 & 0,26 & - & 1,00 & 0,20 & - \\
\hline VAN ROS, VAN SANDE (41) & Agargelelektrophorese & 0,98 & 0,36 & 160 & 1,11 & - & 27 & 0,99 & - & 75 & 0,91 & 一 & 58 \\
\hline FERRIS und Mitarbeiter (42) & $\begin{array}{l}\text { Acrylamidgelelektro- } \\
\text { phorese }\end{array}$ & 1,01 & 0,37 & 182 & 1,26 & 0,34 & 30 & 1,08 & 0,36 & 83 & 0,83 & 0,32 & 69 \\
\hline AUGENER (43) & $\begin{array}{l}\text { Radiale Immuno- } \\
\text { diffusion }\end{array}$ & - & - & - & 1,60 & - & 26 & - & - & - & - & - & - \\
\hline BECKER und Mitarbeiter (29) & $\begin{array}{l}\text { Radiale Immunodif- } \\
\text { fusion }\end{array}$ & - & - & 一 & 1,67 & 0,35 & 26 & 2,36 & 0,73 & 95 & 1,89 & 0,60 & 68 \\
\hline FAUL (44) & $\begin{array}{l}\text { Trübungstest } \\
\text { (immunologisch) }\end{array}$ & 0,91 & 0,40 & 80 & 0,92 & 0,39 & 23 & 1,01 & 0,38 & 26 & 0,82 & 0,44 & 27 \\
\hline & Peroxydase- & 0,93 & 0,38 & 80 & 1,01 & 0,39 & 23 & 0,99 & 0,37 & 26 & 0,80 & 0,40 & 27 \\
\hline Eigene Ergebnisse & $\begin{array}{l}\text { Peroxydase- } \\
\text { Radiale Immuno- } \\
\text { diffusion }\end{array}$ & 0,94 & 0,30 & $\underline{161}$ & $\begin{array}{l}1,00 \\
1,72\end{array}$ & $\begin{array}{l}0,32 \\
0,59\end{array}$ & $\begin{array}{l}47 \\
47\end{array}$ & $\begin{array}{l}0,98 \\
2,37\end{array}$ & $\begin{array}{l}0,27 \\
0,63\end{array}$ & $\begin{array}{l}65 \\
65\end{array}$ & $\begin{array}{l}0,81 \\
1,93\end{array}$ & $\begin{array}{l}0,28 \\
0,66\end{array}$ & $\begin{array}{l}49 \\
49\end{array}$ \\
\hline
\end{tabular}


für die radiale Immunodiffusion notwendige Typendifferenzierung bei niedriger Peroxydaseaktivität in der Regel nicht gelingt. Artefizielle Hämoglobinbeimengungen zum Serum beeinflussen die Ergebnisse beider Verfahren. Für wissenschaftliche Fragestellungen erscheint der kombinierte Einsatz beider Methoden sinnvoll: durch den Vergleich der jeweils gewonnenen Werte können Hämoglobinbindungsanomalien des Haptoglobins aufgedeckt werden.

\section{Danksagung}

Für die Uberlassung von Reinproteinen der 3 Haptoglobintypen danken wir Herrn Dr. H. G. Schwick (Behring-Werke, Marburg).

Herrn Priv.-Doz. Dr. W. Schneider, Vorsteher d. Abt. f. Transfusionswesen an der Chir. Univ. Klin. Tübingen, danken wir für die freundliche Uberlassung der Seren.

Fräulein G. BEHrmanN danken wir für zuvcrlässige und wertvolle technische Assistenz.

\section{Literatur}

1. Laurell, C.-B. und M. Nyman, Blood 12, 493 (1957). - 2. Merril, D. A., C. H. Kirkpatrick, W. E. C. Wilson und C. M. Riley, Proc. Soc. Exper. Biol. Med., N. Y. 116, 748 (1964). 3. Bock, H. E., H. F. v. Oldershausen, F. W. Aly und M. KreBs, Blut 19, 232 (1969). - 4. Kallai, L., M. Keler-BaCoKa, K. Blazevic und S. KNEZEvic, Gastroenterologia 105, 27 (1966). 5. Prellwitz, W., C. H. Hammar und J. Dudeck, Dtsch. med. Wschr. 93, 1277 (1968). - 6. JaYLe, M.-F. und G. Boussrer, Expos. Ann. Biochim. Med., 17, 157 (1955). - 7. Braun, H. J., Habilitationsschrift, Tübingen 1969. - 8. JAYLE, M.-F., Ann. biol. clin. 1, 2 (1943). - 9. Mancini, G., J. P. Vaerman, A. O. Carbonara und J. F. Heremans, Prot. Biol. Fluids, 11th Coll. pp 370 (1963), Elsevier Pub. Comp., Amsterdam (1964). 10. Aly, F. W. und H. J. Braun, Klin. Wschr. 46, 385 (1968). 11. Braun, H. J. und F. W. Axy, Clin. Chim. Acta, Amsterdam 26, 588 (1969). - 12. Alx, F. W. und H. J. Braun, Prot. Biol. Fluids, 18th Coll. pp. 361 (1970), Elsevier Pub. Comp., Amsterdam (1971). - 13. NrmaN, M., Scand. J. Clin. Laborat. Invest. 11, Suppl. 39, 1 (1959). - 14. Nrman, M., Clin. Chim. Acta, Amsterdam 3, 111 (1958). - 15. BECKER, W., W. RAPP, H. G. SCHWICK und K. STöRIKo, diese Z. 6, 113 (1968). - 16. Smrthres, O., Biochem. J. 71, 585 (1959). - 17. Cohnen, G. und D. PaAr, diese Z. 7, 63 (1969). 18. JAYLe, M.-F., Compt. rend. Acad. Sc. 211, 574 (1950). 19. Herman-Boussier, G., J. Moretti und M.-F. Jayle, Bull. Soc. chim. biol. 42, 837 (1960). - 20. BRAUNITZER, G., R. GEHRing-Müller, N. HilschmanN, K. Hilse, G. Hobom, V. RudLOFF und B. WITTMANN-Limbold, Hoppe-Seyler's Z. physiol. Chem. 325, 283 (1961). - 21. BLACK, J. A. und G. H. Drxon, Nature, London 218, 736 (1968). - 22. Gordon, S., H. CLEVE und A. G. Brarn, Proc. Soc. Exper. Biol. Med., N. Y. 127, 52
(1968). - 23. BlaCk, J. A., G. F. Q. Chan, C. L. Hew und G. H. Dixon, Canad. J. Biochem. 48, 123 (1970). - 24. Cloarec, L. und J. Moretri, Compt. rend. Acad. Sc. D 262, 2081 (1966). 25. Hamaguchi, H., Proc. Japan. Acad. 44, 733 (1968). - 26. Javid, J., Vox. Sang. 10, 320 (1965). - 27. Smith, H. und J. A. Owen, Biochem. J. 78, 723 (1961). - 28. OgawA, A. und K. Kawamura, Proc. Japan. Acad. 42, 413 (1966). - 29. Becker, W., N. Heimburger, H. G. Schwick und K. Störiko, 7. Int. Congr. Klin. Chem., Genf (1969). - 30. Barnicot, N. A., J. P. Garlick und D. F. Roberts, Ann. Hum. Genet. 24, 171 (1960). - 31. Gottrieb, A., N. Wisch und J. Ross, Blood 21, 129 (1963). 32. Beuing, H., H. Cleve und H. Deicher, Z. Hyg. 151, 291 (1965). - 33. Shim, B.-S., T.-H. LeE und Y.-S. KANG, Nature, London 207, 1264 (1965). - 34. KorNGOLD, L., Immunochemistry 2, 103 (1965). - 35. EichmanN, K., D. Deicher und H. Cleve, Humangenetik 2, 271 (1966). - 36. JAVID, J. und W. Ynngling, J. Clin. Invest. 47, 2290 (1968). - 37. Allison, A. C. und W. ap REES, Brit. med. J. 1957/II, 1137. - 38. BAYANISioson, P. S., J. Louch, H. E. Sutton, J. V. Neel, S. L. Horne und H. Gershowitz, Amer. J. Hum. Genet. 14, 210 (1962). 39. Whittaker, M., Amer. J. Clin. Path. 50, 454 (1968). - 40. Allison, A. C., Proc. Roy. Soc. Med. 51, 641 (1958). - 41. van Ros, G. und M. van Sande, Clin. Chim. Acta, Amsterdam 10, 62 (1964). - 42. Ferris, Th. G., R. E. Easterling, K. J. Nelson und R. E. Budd, Amer. J. Clin. Path. 46, 385 (1966). 43. Augener, W., Prot. Biol. Fluids 12th Coll. pp. 363 (1964), Elsevier Pub. Comp., Amsterdam (1965). - 44. FAuL, J., Inaugural-Dissertation, Freiburg 1967. - 45. GABL, F. und H. HUBER, Wien. klin. Wschr. 77, 1037 (1965).

Privatdozent Dr. H. J. Braun Abt. Innere Medizin II

Privatdozent Dr. F. W. Aly Medizinische Universitätsklinik 74 Tübingen Otfried-Müller-Straße 